\title{
SEISMIC RESPONSE OF TORSIONALLY IRREGULAR SINGLE STORY STRUCTURES
}

\author{
Eu Ving Au ${ }^{1}$, Gregory MacRae ${ }^{2}$, Didier Pettinga ${ }^{3}$, Bruce Deam ${ }^{4}$, \\ Vinod Sadashiva $^{5}$ and Hossein Soleimankhani ${ }^{6}$
}

(Submitted July 2018; Reviewed November 2018; Accepted January 2019)

\begin{abstract}
Impulse ground motions are applied to single story structures with different in-plane wall strength and stiffness, rotational inertia, and out-of-plane wall stiffness to obtain the dynamic response considering torsion. A simple hand method to evaluate the impulse response is developed. It is shown that the median increase in response of the critical component considering torsion from many earthquake records is similar to that from impulse records. Using this information, a simple design methodology is proposed which enables the likely earthquake response of critical elements considering torsion to be obtained from building analyses not considering torsion. A design example is also provided.
\end{abstract}

\section{INTRODUCTION}

For elastic structures, torsion is caused by stiffness eccentricities relative to the centre of mass. Such an eccentricity occurs from differences in stiffness between lateral-force resisting elements [1]. There is a significant body of research on the asymmetric response of these structures which has been summarised by Rutenburg [2] and De Stefano and Pintucchi [3]. Rutenburg [2] has also summarised several works have on the nonlinear response of asymmetric structures to bidirectional excitation. De Stefano and Pintucchi [3] reviewed the use of passive energy dissipating devices to decrease detrimental effects of torsion.

For yielding structures, such as those which are subject to strong earthquake motions, torsional deformations may be accentuated by strength eccentricities. In Figure 1(a), a plan of a structure with constant stiffness in both in-plane walls and the mass centroid at the centre of the structure is shown. Before yielding occurs, both walls have the same deformation due to north-south translational shaking. If Wall 1 is weaker than Wall 2 , then deformation at Wall 1 is likely to be significantly greater than that at Wall 2, as shown in Figure 1(b). This torsional effect must be considered to correctly evaluate wall demands for design. Methods to estimate the torsional effect are therefore required.

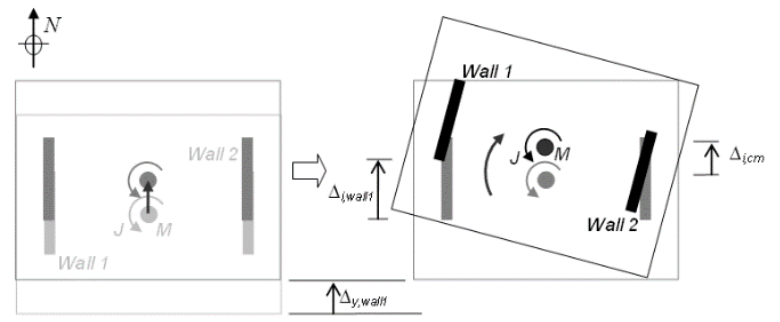

(a)

(b)

Figure 1: Inelastic torsional mechanism.
Perhaps the simplest and easily applied concept for estimating torsional response in yielding structures was developed by Paulay [4]. According to Paulay, rotational inertia effects are ignored and the displacement of the centre of mass is found to be the same irrespective of whether or not torsion occurs. For example, consider the simple structure of Figure 1 where there are no out-of-plane walls (i.e. it is a torsionally unrestrained system), the walls have the same stiffness and are elastoplastic, and the yield strength of Wall 2 is greater than that of Wall 1 . Here, when both walls are pushed to the yield displacement of Wall $1, \Delta_{y}$,Wall, then yielding occurs in that wall and Wall 2 stops moving further. At the peak displacement response, the centre of mass has a total displacement, $\Delta_{u, c m}$, which is equal to

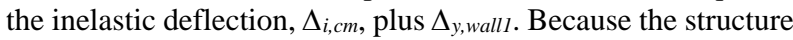
is symmetric, the total displacement of the critical wall, $\Delta_{u, \text { crit }}$, is given by Equation 1 as a result of geometric considerations.

$\Delta_{u, c r i t}=\Delta_{y, \text { wall } 1}+2 \Delta_{i, c m}$

For more general cases, where the in-plane walls have different stiffness and the centre of mass is not at the centre of the structure, the response of the structure could be estimated by first ignoring torsion to give $\Delta_{u, c m}$, and then using a static push through the centre of mass considering torsion to the same displacement, $\Delta_{u, c m}$, to finally obtain $\Delta_{u, c r i t}$.

While Paulay's concept is simple, it does not consider the dynamic effect of rotational inertia, or out-of-plane walls. Paulay [5, 6] showed by means of dynamic inelastic time history analysis with rotational inertia effects included, that the rotational inertia reduces the propensity for twist about the vertical axis. It was also shown that the time of peak rotation in the structure was often not the same as that of the peak displacement in the critical element. Further investigation by Castillo [7] followed the concepts of Paulay, resulting in the recommendation that the centre of strength, rather than the centre of stiffness, as commonly used in earlier studies, should be as close as possible to the centre of mass.

\footnotetext{
${ }^{1}$ Ruamoko Solutions, Christchurch, New Zealand

${ }^{2}$ Associate Professor, Department of Civil and Natural Resource Engineering, University of Canterbury, Christchurch, New Zealand

${ }^{3}$ Holmes Consulting, Christchurch, New Zealand

${ }^{4}$ Ministry of Business, Innovation \& Employment, Christchurch, New Zealand

5 GNS SCIENCE, Lower Hutt, New Zealand

${ }^{6}$ Corresponding Author, PhD Candidate, Department of Civil and Natural Resource Engineering, University of Canterbury, Christchurch, New Zealand, (hossein.soleimankhani@pg.canterbury.ac.nz)
} 
Similar findings were published by Myslimaj and Tso [8], where the focus was on achieving a stable (or limited) torsional response through explicit design to locate the centres of strength and stiffness evenly either side of the centre of mass. While the emphasis is on strength here, these authors have argued that in real structures strength is often proportional to stiffness. Therefore, by satisfying the strength criteria, the stiffness criteria is also satisfied. In these cases, the recommendations are appropriate for elastic, or near elastic, response where stiffness is important, as well as for very ductile response where strength is important. While methods of this type are appropriate for design, due to various planned and accidental reasons, it may not be possible to follow such criteria. Also, in some structural types while strength may be related to stiffness for a particular member, the structure may contain a number of member or system types where strength and stiffness in general are not related. Guidance for these cases are also required.

Recent work by Trombetti et al. [9] and Sommer and Bachmann [10] assumed that inelastic diaphragm rotations are adequately and conservatively represented by elastic results. In both studies, solutions derived from elastic dynamic theory were found, and compared to results from non-linear time history analyses. As highlighted by Pettinga et al. [11], such solutions do not allow an identification of the yield mechanism that leads to peak asymmetric response, making them somewhat unsuitable to incorporation within a capacity design context.

The N2 method [12] was extended by Fajfar et al. [13] to include application for asymmetric building structures. This method considers dynamic effects using inelastic spectra combined with a static 3D pushover analysis. The major limitation of this method is that it involves converting a multidegree of freedom (MDOF) system into an equivalent singledegree of freedom (SDOF) system to calculate the demands from response spectra. This does not apply well to irregular structures where higher modes have a greater contribution.

The study by Castillo [7] clearly demonstrated the effect of the twist restraint present within the system due to the rotational mass inertia. Pettinga et al. [11] proposed a method to predict the asymmetric inelastic response of simple single-storey systems, which included the quantification of "dynamic rotational stiffness" due to the rotational inertia. This development was derived from an energy balance, and was presented for a set of systems with varying asymmetric and restraint conditions, subjected to half-sine pulses with different magnitudes and periods.

Recently Beyer [14] developed a method to estimate the inelastic response of torsionally restrained structures using an effective torsional stiffness within a displacement-based design context. While the method does not include an allowance for the mass rotational inertia, it was found to give sufficient estimates of displacement demand for design purposes for the cases considered.

Codes around the world generally account for torsional effects in the following ways:

1. For accidental torsional effects, due to uncertainty about the actual centre of mass location, the actual stiffness and strength on any story, the nominal centre of mass is artificially moved, usually by some fraction of the plan dimension, to increase the demands on the frame under consideration. This procedure is sometimes also considered to account for torsional ground motions.

2. For structures which are defined as torsionally irregular, use of some simple analysis technique is not permitted as it is considered that they may not predict the response appropriately. Often a three-dimensional elastic modal analysis is carried out for these structures, even though only three-dimensional nonlinear analyses are likely to capture the inelastic torsional response.
It may be seen from the discussion above that there is a need for a simple method to understand the total response of general structures which may deform torsionally during earthquake motion. This method should consider rotational inertia, out-ofplane walls, and both high and low ductility demands. It should be appropriate for structures with both high and low levels of torsional sensitivity, for different assumptions related to strength and stiffness dependency, and it should have a strong fundamental (rather than empirical) basis. The method should also be able to be transformed into a design/assessment method so that important effects of torsion can be anticipated and applied appropriately to design methods to mitigate these effects.

This paper is a step toward satisfying this need. In particular, Paulay's [4] work in torsion is firstly extended for single story structures considering dynamic effects using impulse loading, or a nonlinear impulse procedure (NIP). Empirical methods based on actual earthquake methods are then used to modify the response of critical elements due to torsion for realistic demand estimation. In particular, answers are sought to the following questions:

1. What is the importance of rotational inertia on the response and does impulse loading adequately represent what is already known in literature?

2. Can a closed-form solution be derived for a single-storey system subject to an impulse based on two-degree-offreedom free vibration concepts?

3. How do various parameters influence the response to impulse and which of these have the greatest effect?

4. What is the applicability of the non-linear impulse procedure (NIP) in the context of a design approach for earthquake excitation?

\section{STRUCTURE AND MODELLING}

The benchmark single story building used in analyses was taken from Castillo [7]. Figure 2 below shows a plan view of the structure. Benchmark parameters used are given in Table 1 below. All analyses in subsequent sections used these parameters, unless stated otherwise. The floor slab was analysed as a perfectly rigid diaphragm.

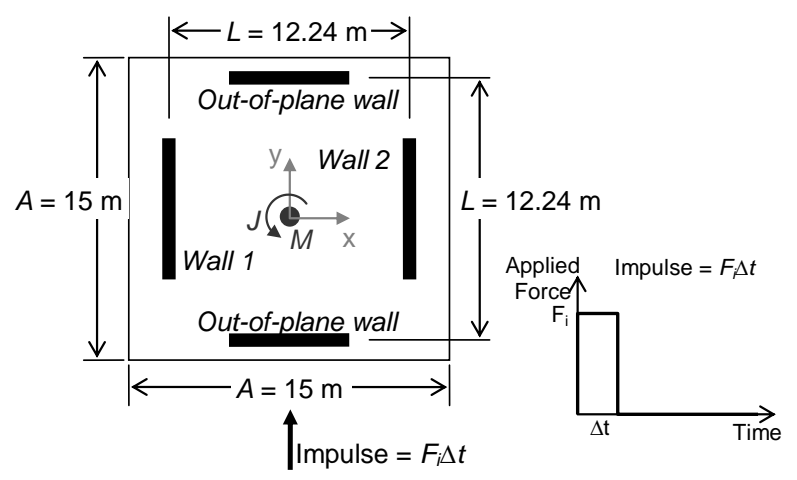

Figure 2: Plan view of single storey benchmark building [7].

The mass of the square diaphragm was represented as both a point mass, $M$, and a rotational mass, $J$, coincident with the centre of the rigid diaphragm. Although these are referred to as masses, herein $M$ and $J$ shall be specified here in terms of weight. The benchmark value for $M$ was $1766 \mathrm{kN}$. The rotational mass for a uniformly distributed mass over a square floor slab is $J_{r}$, given in Equation 2, where $A$ is the length of floor diaphragm and $\hat{m}$ is the weight per unit area of floor diaphragm. Since the rotational mass, $J$, corresponding to the point mass, $M$, is dependent on the distribution of the mass over floor and not all floors with the mass of $M$ have the rotational mass of $J_{r}$, as computed in Equation 2, the benchmark value was 
arbitrarily taken as one-third of this, to consider a lower level of rotational mass that amplifies the torsional response.

$$
\begin{aligned}
J_{r}=\frac{\widehat{m} A^{4}}{6}=\frac{M A^{2}}{6} & =\frac{1766 k N \times(15 m)^{2}}{6} \\
& =66225 k N-m^{2}
\end{aligned}
$$

Table 1. Benchmark parameters.

\begin{tabular}{ccc} 
Parameter & Symbol & Benchmark value \\
\hline Mass & $M$ & $1766 \mathrm{kN}$ \\
Rotational mass & $J$ & $0.333 J_{r}$ \\
Wall 1 stiffness & $K_{l}$ & $14212 \mathrm{kN} / \mathrm{m}$ \\
Wall 2 stiffness & $K_{2}$ & $K R \times K_{l}$ \\
Wall 1 yield strength & $F_{y l}$ & $(K R=1)$ \\
Wall 2 yield strength & $F_{y 2}$ & $220.3 \mathrm{kN}$ \\
& & $\left(S R \times F_{y 2}\right.$ \\
Out-of-plane wall stiffness & $K_{\text {out }}$ & $K R_{\text {out }} \times K_{l}$ \\
Bilinear factor & $r$ & $\left(K R_{\text {out }}=0\right)$ \\
& & 0.0001
\end{tabular}

The benchmark parameters for Wall 1 were stiffness, $K_{l}$, of $14,212 \mathrm{kN} / \mathrm{m}$ and yield strength, $F_{y l}$, of $220 \mathrm{kN}$. The properties of Wall 2 were then specified as a ratio of Wall 1's properties. An in-plane wall stiffness ratio, $K_{R}$, of 1.0 and a strength ratio, $S R$, of 1.36 (i.e. $F_{y 2}=1.36 \times 220.3=300 \mathrm{kN}$ ) were adopted as benchmark values. A bilinear hysteresis loop with a post-yield stiffness ratio, $r$, of 0.0001 was chosen. This was to provide post-yield stability for the computer model and approximate elastic perfectly plastic response [13]. This was considered to provide a conservative estimate of displacement for most real structures.

In analyses including out-of-plane walls (i.e. $K_{\text {out }}>0$ ), the walls were modelled as an equivalent rotational spring located at the centre of mass. Herein, out-of-plane wall stiffness is specified as a ratio, $K R_{\text {out }}$, of Wall 1's benchmark stiffness of 14,212 $\mathrm{kN} / \mathrm{m}$. The rotational stiffness, $k_{r}$, calculated in Equation 3 corresponds to an out-of-plane wall stiffness ratio, $K R_{\text {out }}$, of 1 ; that is, two out-of-plane walls of identical stiffness to Wall 1. For all analyses it was assumed that these walls would remain elastic throughout the entire response. No out-of-plane walls $\left(K R_{\text {out }}=0\right)$ was adopted as the benchmark.

$k_{r}=\frac{K_{\text {out }} L^{2}}{2}=\frac{14212.2 \mathrm{kN} / \mathrm{m} \times(12.24 \mathrm{~m})^{2}}{2}$

$$
=1,064,619 \mathrm{kNm}
$$

All NIP analyses were performed in RUAUMOKO2D (Carr, 2005) using Newmark's constant average acceleration integration method. The impulse, $F_{i} \Delta t$, shown in Figure 2, was a constant force over one time-step, $\Delta \mathrm{t}$, of $0.001 \mathrm{~s}$. Damping was ignored in all impulse analyses to allow a simple closed form solution to be developed. The impulse force magnitude was that required to produce a benchmark translational ductility demand of 5 for the building with no twist about the vertical axis. This was found to be $105,210 \mathrm{kN}$, and produced an ultimate displacement of $77.5 \mathrm{~mm}$, five times the yield displacement of $15.5 \mathrm{~mm}$.

\section{ROTATIONAL INERTIA EFFECT}

While many authors have shown that rotational inertia may significantly affect the response of a torsionally unrestrained system, in the context of NIP this is not immediately obvious based on the argument below.

An impulse imparts a fixed amount of kinetic energy into the system. This energy can either be stored elastically or dissipated by the yielding element. Consider a system in which only one wall yields. It may be hypothesised that systems with and without rotational mass should have the same peak displacement, because energy can only be dissipated in the yielding wall.

The dashed line in Figure 3(a) shows that the response of the weaker wall with significant rotational inertia is less than the response with no rotational inertia. This wall defined as the critical wall in this study. Here Wall 1 can yield in both the positive and negative displacement directions, and this is referenced to as bi-directional yielding. This decrease in response is in conflict with the idea presented in the preceding paragraph. Further investigation of the response indicated that the reason for the difference is a result of higher mode effects. In the absence of rotational inertia, the critical wall only yields in the loading direction. When rotational inertia is included (i.e. $J>0$ ), yielding occurs in two directions using up the structural energy such that peak response decreases.

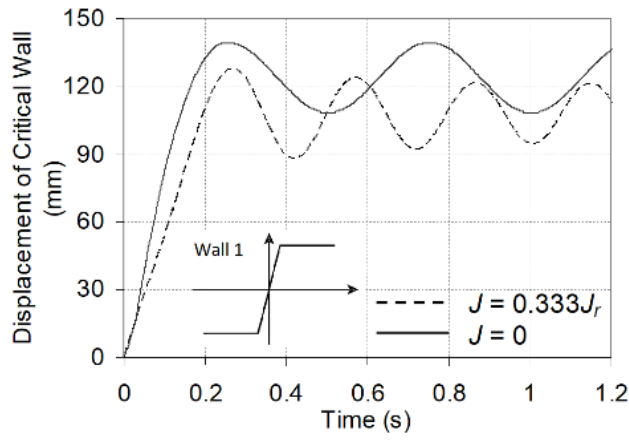

(a) Wall 1 bi-directional yielding

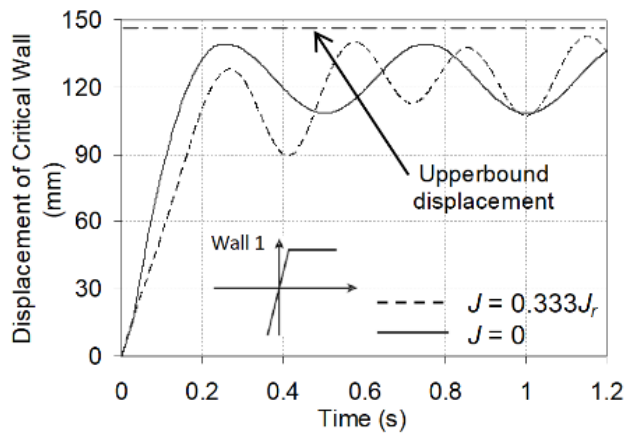

(b) Wall 1 uni-directional yielding

\section{Figure 3: Effect of J on critical wall (Wall 1) response (Wall 2 elastic: $S R=\infty$ ).}

Figure 3(b) shows the response where the critical wall, Wall 1, is only permitted to yield in the positive loading direction, and it is made elastic (non-yielding) in the unloading direction. This is referred to as uni-directional yielding. Here the response with no rotational inertia $(J=0)$ is identical to that in Figure 3(a) indicating that yielding in the unloading direction does not occur in this case. The displacement of the structure with rotational inertia in Figure 3(b) is significantly increased compared to Figure 3(a), because in Figure 3(b) yielding cannot occur in the unloading direction. An upper bound displacement line is shown in the figure. This is the displacement that would 
occur if all of the energy imparted to the system by the impulse were dissipated by yielding in the critical wall. The impulse, $F . \Delta t$, equals $M . \Delta V$ and the kinetic energy can be obtained using this formula, $E_{k}=1 / 2 M V^{2}$. The energy absorption capacity of a yielding wall with elastic perfectly plastic forcedisplacement relationship is $E_{P}=F_{y} \Delta_{y} / 2+F_{y}\left(\Delta_{u}-\Delta_{y}\right)$. The upper bound displacement $\Delta_{U}$ is obtained by assuming $E_{P}=E_{k}$. Neither oscillator attains this upper bound displacement because, at the time of the peak displacement of the critical wall, either the mass at the centre of the structure has a velocity (and kinetic energy), the other wall has some force (and hence potential energy), or both.

\section{TWO DEGREE OF FREEDOM MODEL}

\section{Development of 2DOF Solution}

A 2DOF analytical model was developed to investigate single storey building torsional response to impulse. Figure 4 shows a plan view schematic of the $2 \mathrm{DOF}$ freedom system adopted for this purpose. The two degrees of freedom are translation, $y$, and rotation, $\theta$, at the centre of mass. To keep this solution as general as possible, allowance for out-of-plane walls was made, given by an equivalent rotational spring of stiffness $k_{r}$.

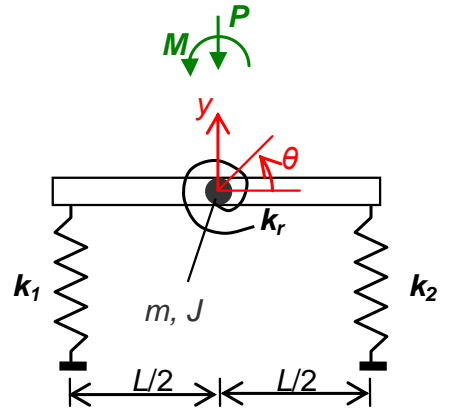

Figure 4: Schematic of general 2DOF system.

The equations of motion for the above system are:

$$
\begin{array}{r}
{\left[\begin{array}{cc}
m & 0 \\
0 & J
\end{array}\right]\left\{\begin{array}{l}
\ddot{y} \\
\ddot{\theta}
\end{array}\right\}+\left[\begin{array}{cc}
k_{1}+k_{2} & \left(k_{2}-k_{1}\right) L / 2 \\
\left(k_{2}-k_{1}\right) L / 2 & k_{r}+\left(k_{1}+k_{2}\right) L^{2} / 4
\end{array}\right]\left\{\begin{array}{l}
y \\
\theta
\end{array}\right\}=} \\
\left\{\begin{array}{c}
-P \\
M
\end{array}\right\}
\end{array}
$$

where $y$ and $\theta$ are two degrees of freedom of the system (Figure 4), $m$ and $J$ are concentrated translational and rotational mass respectively and $k_{1}$ and $k_{2}$ are translational stiffness in the direction of interest and $k_{r}$ is the equivalent rotational stiffness due to out-of-plane walls stiffness (i.e. Equation (3)). According to Figure 2, $L$ is the distance between walls.

This set of equations can be solved to give:

$$
\begin{aligned}
& y=A \Phi_{1 y} \cos \left(\omega_{1}(\right.\left.\left.t-t_{0}\right)\right)+B \Phi_{1 y} \sin \left(\omega_{1}\left(t-t_{0}\right)\right) \\
&+\Phi_{1 y} \frac{P_{y}^{*}}{K_{y}^{*}}+C \Phi_{2 y} \cos \left(\omega_{2}\left(t-t_{0}\right)\right) \\
&+D \Phi_{2 \theta} \sin \left(\omega_{2}\left(t-t_{0}\right)\right)+\Phi_{2 y} \frac{P_{\theta}^{*}}{K_{\theta}^{*}} \\
& \theta=A \Phi_{1 \theta} \cos \left(\omega_{1}\left(t-t_{0}\right)\right)+B \Phi_{1 \theta} \sin \left(\omega_{1}\left(t-t_{0}\right)\right) \\
&+\Phi_{1 \theta} \frac{P_{y}^{*}}{K_{y}^{*}}+C \Phi_{2 \theta} \cos \left(\omega_{2}\left(t-t_{0}\right)\right) \\
&+D \Phi_{2 \theta} \sin \left(\omega_{2}\left(t-t_{0}\right)\right)+\Phi_{2 \theta} \frac{P_{\theta}^{*}}{K_{\theta}^{*}}
\end{aligned}
$$

$$
\begin{aligned}
\dot{y}=-A \Phi_{1 y} \omega_{1} \sin & \left(\omega_{1}\left(t-t_{0}\right)\right)+B \Phi_{1 y} \omega_{1} \cos \left(\omega_{1}\left(t-t_{0}\right)\right) \\
& -C \Phi_{2 y} \omega_{2} \sin \left(\omega_{2}\left(t-t_{0}\right)\right) \\
& +D \Phi_{2 y} \omega_{2} \cos \left(\omega_{2}\left(t-t_{0}\right)\right) \\
\dot{\theta}=-A \Phi_{1 \theta} \omega_{1} \sin & \left(\omega_{1}\left(t-t_{0}\right)\right)+B \Phi_{1 \theta} \omega_{1} \cos \left(\omega_{1}\left(t-t_{0}\right)\right) \\
& -C \Phi_{2 \theta} \omega_{2} \sin \left(\omega_{2}\left(t-t_{0}\right)\right) \\
& +D \Phi_{2 \theta} \omega_{2} \cos \left(\omega_{2}\left(t-t_{0}\right)\right)
\end{aligned}
$$

where:

$$
\begin{gathered}
K_{y}^{*}=\Phi_{1 y}^{2}\left(k_{1}+k_{2}\right)+\Phi_{1 y} \Phi_{1 \theta}\left(k_{2}-k_{1}\right) L \\
+\Phi_{1 \theta}^{2}\left(k_{1}+k_{2}\right) \frac{L^{2}}{4}+\Phi_{1 \theta}^{2} k_{r} \\
K_{\theta}^{*}=\Phi_{2 y}^{2}\left(k_{1}+k_{2}\right)+\Phi_{2 y} \Phi_{2 \theta}\left(k_{2}-k_{1}\right) L \\
+\Phi_{2 \theta}^{2}\left(k_{1}+k_{2}\right) \frac{L^{2}}{4}+\Phi_{2 \theta}^{2} k_{r} \\
P_{y}^{*}=-\Phi_{1 y} P+\Phi_{1 \theta} M \\
P_{\theta}^{*}=-\Phi_{2 y} P+\Phi_{2 \theta} M
\end{gathered}
$$

$\omega_{i}$ and $\Phi_{i}$ are the natural frequencies and mode shapes of the system respectively and $A, B, C$ and $D$ are arbitrary constants. $P$ and $M$ are the external load are applied to the system in the $y$ and $\theta$ directions.

From the initial conditions: $y\left(t_{0}\right)=y_{0}, \dot{y}\left(t_{0}\right)=\dot{y}_{0}, \theta\left(t_{0}\right)=$ $\theta_{0}$ and $\dot{\theta}\left(t_{0}\right)=\dot{\theta}_{0}$, the constants are:

$$
\begin{aligned}
& A=\frac{\theta_{0} \Phi_{2 y}-y_{0} \Phi_{2 \theta}}{\left(\Phi_{2 y} \Phi_{1 \theta}-\Phi_{1 y} \Phi_{2 \theta}\right)}-\frac{P_{y}^{*}}{K_{y}^{*}} \\
& B=\frac{\dot{\theta}_{0} \Phi_{2 y}-\dot{y}_{0} \Phi_{2 \theta}}{\omega_{1}\left(\Phi_{2 y} \Phi_{1 \theta}-\Phi_{1 y} \Phi_{2 \theta}\right)} \\
& C=\frac{y_{0} \Phi_{1 \theta}-\theta_{0} \Phi_{1 y}}{\left(\Phi_{2 y} \Phi_{1 \theta}-\Phi_{1 y} \Phi_{2 \theta}\right)}-\frac{P_{\theta}^{*}}{K_{\theta}^{*}} \\
& D=\frac{\dot{y}_{0} \Phi_{1 \theta}-\dot{\theta}_{0} \Phi_{1 y}}{\omega_{2}\left(\Phi_{2 y} \Phi_{1 \theta}-\Phi_{1 y} \Phi_{2 \theta}\right)}
\end{aligned}
$$

The frequencies, $\omega_{i}$, and mode shapes, $\Phi_{i}$, are found from a free-vibration analysis $(M=0, P=0)$ where the fundamental frequency, $\omega_{1}$, is the lowest one obtained using the negative sign in Equation 17.

$$
\begin{aligned}
& \omega^{2}=\frac{1}{2 m J}\left[m\left(k_{r}+\frac{\left(k_{1}+k_{2}\right) L^{2}}{4}\right)+J\left(k_{1}+k_{2}\right)\right] \pm \\
& \frac{1}{2 m J}\left[\sqrt{\left(m\left(k_{r}+\frac{\left(k_{1}+k_{2}\right) L^{2}}{4}\right)-J\left(k_{1}+k_{2}\right)\right)^{2}+m J L^{2}\left(k_{2}-k_{1}\right)^{2}}\right]
\end{aligned}
$$

$\left\{\Phi_{i}\right\}=\left\{\begin{array}{c}1.0 \\ \left.\frac{2 m \omega_{i}^{2}}{L\left(k_{2}-k_{1}\right)}-\frac{2\left(k_{1}+k_{2}\right)}{L\left(k_{2}-k_{1}\right)}\right\}\end{array}\right.$

The displacement of each wall in this case can then be calculated by summing the translational response at the centre of mass with the additional displacement due to rotation, such that:

$y_{\text {Wall } 2}=y-\frac{\theta L}{2}$
$y_{\text {Wall } 2}=y+\frac{\theta L}{2}$

The above equations are an exact analytical solution for the 2DOF system. The full derivation is given in Au [15]. 
This solution, with specified loading, can be modified to consider elastic free vibration, yielding of the first wall, and yielding of the second wall. Specifying a rotational stiffness also allows out-of-plane walls to be considered.

During the elastic portion of the free vibration response, the 2DOF system has $M=0$ and $P=0$. Both walls will have their respective elastic stiffness of $K_{l}$ and $K_{2}$, and the system will have the following initial conditions: $t_{0}=t_{1}, y_{0}=y_{1}, \dot{y}_{0}=\dot{u}_{1}, \theta_{0}=$ $\theta_{1}$ and $\dot{\theta}_{0}=\dot{\theta}_{1}$. If the impulse duration is very small, as it tends to be in practice, then $t_{0}=0, y_{0}=0, \dot{y}_{0}=\dot{u}_{i}, \theta_{0}=0$ and $\dot{\theta}_{0}=$ 0 where $\dot{u}_{i}$ is the velocity imparted to the structure by the impulse.

At yielding of the first wall, Wall 1, its stiffness becomes its postelastic stiffness, $r K_{l}$. The yield force in Wall 1 is then represented as a constant force $P=F_{y l}$, and moment, $M=F_{y l} L / 2$, applied at the centre of mass as shown in Figure 5. The initial conditions are then the final conditions of the elastic response, namely $t_{0}=$ $t_{y}, y_{0}=\Delta_{y}, \dot{y}_{0}=\dot{u}_{y}, \theta_{0}=\theta_{y}$ and $\dot{\theta}_{0}=\dot{\theta}_{y}$. The rotational terms, $\theta_{0}$ and $\dot{\theta}_{0}$, will both be zero if $K_{1}=K_{2}$.

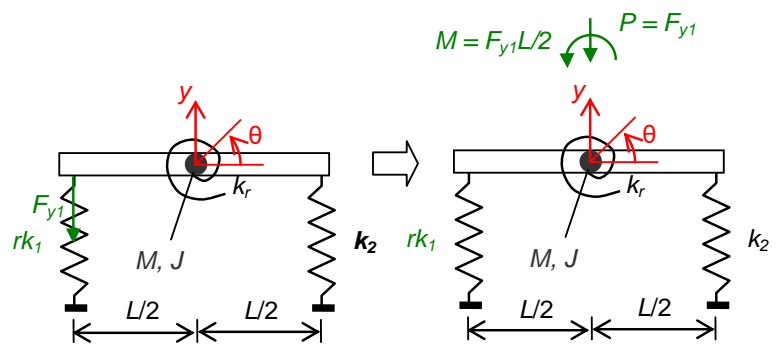

Figure 5: 2DOF system for one-wall-yielding.

When the second wall, Wall 2, yields, it now has been reduced to its post-elastic stiffness, $r K_{2}$. Similarly, the yield force at Wall 2 can be represented by $P=\left(F_{y 1}+F_{y 2}\right)$ and $M=\left(F_{y 1}-F_{y 2}\right) L / 2$ at the centre of mass. The initial conditions are taken as the values when the second wall reaches its yield displacement.

It should be noted that there is no simple closed form solution for the peak response but the solution can be obtained using simple numerical methods. For layouts with different numbers of walls and floor plans, a revised and more complex solution may be developed.

\section{Generality of 2DOF Solution}

Figure 6 shows the response of a system with Wall 1 yielding, Wall 2 fully elastic, and a rotational mass of $0.333 \mathrm{~J}_{r}$. Included on the figure is both the response from the analytical $2 \mathrm{DOF}$ solution and from computer time history analysis RUAUMOKO-2D [16]. Both plots show identical results up to the peak displacement of the yielding wall therefore validating the $2 \mathrm{DOF}$ solution. Later, the two results begin to deviate because the equations developed do not consider unloading after yield, however this does not affect the predicted peak wall displacement.

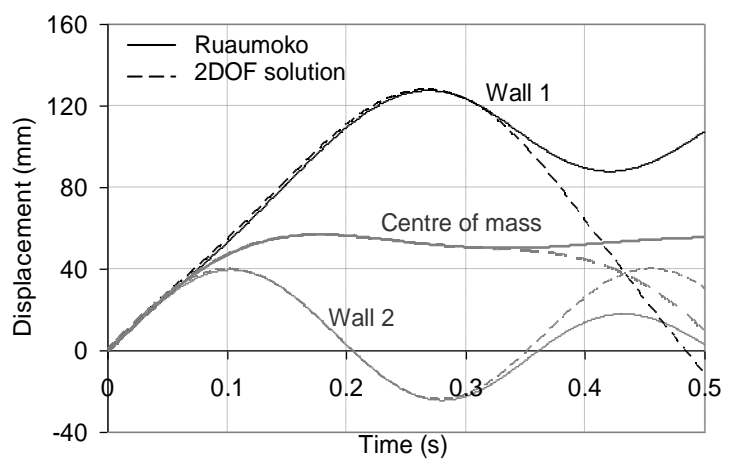

Figure 6: Displacement response with one yielding wall (Wall 2 elastic: $S R=\infty$ ).

Figure 7 shows the response when the second wall is given a strength ratio of 1.36 and is allowed to yield. It shows that the second wall reaches its peak displacement before Wall 1 and that the 2DOF solution is valid up until this point. However, because the peak displacement of Wall 1 occurs after this, its peak response is not predicted well by the 2DOF solution because Wall 2 is unloading and this is not captured in the simple analytical solution. In this case, the peak displacement is overestimated by $12 \%$. The $2 \mathrm{DOF}$ solution would therefore be conservative for design.

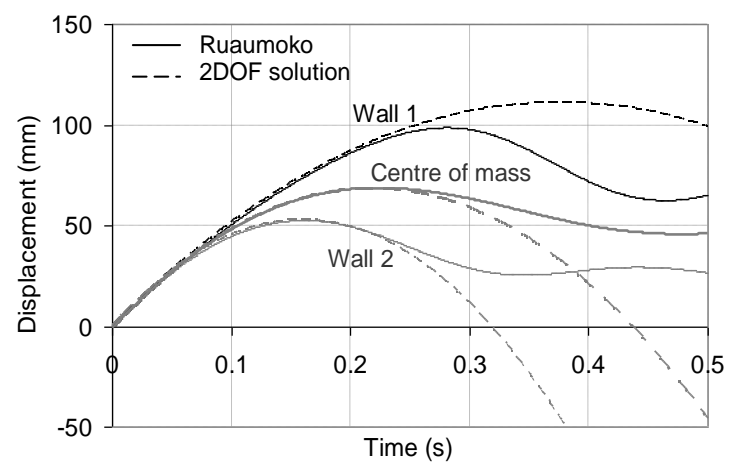

Figure 7: Displacement response with two walls yielding.

When out-of-plane walls are included in the analysis, the 2DOF solution gives a better approximation of the peak response of both walls. This is shown in Figure 8, where again a strength ratio of 1.36 is used and Wall 2 reaches its peak displacement first. The figure shows that for systems with sufficiently high out-of-plane wall stiffness the peak displacement of Wall 1 can also be predicted reasonably accurately.

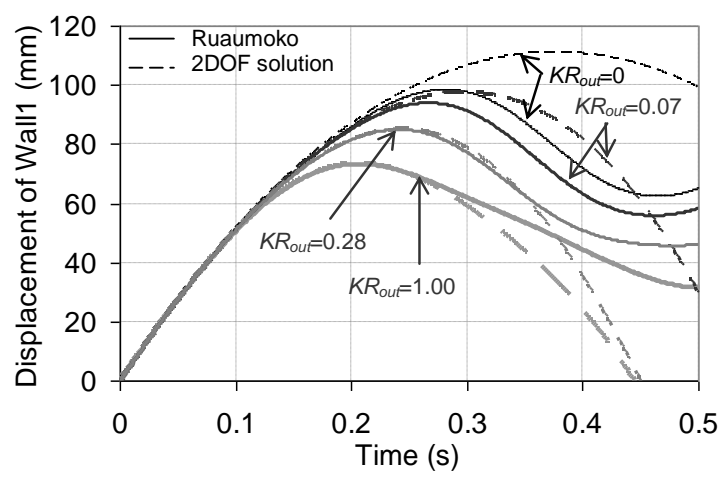

Figure 8: Critical wall displacement with two walls yielding and out-of-plane walls. 
For an out-of-plane wall stiffness equal to Wall 1's stiffness $\left(K R_{\text {out }}=1\right)$ the approximation is independent of the wall strength ratio as shown in Figure 9. That is, the 2DOF solution will still give reasonable predictions for both walls [16].

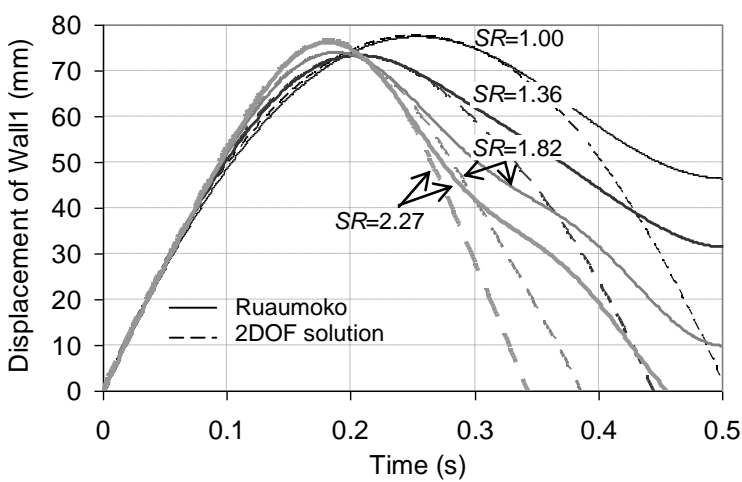

Figure 9: Critical wall displacement with two walls yielding and out-of-plane walls (KRout=1.0).

\section{Solution Stability}

The solution was found to become unstable when the strength and stiffness of both walls were identical and when rotational mass is zero - effectively resulting in a SDOF system. While the second degree of freedom could be condensed out, a rotational mass of $1 \mathrm{kN}-\mathrm{m}^{2}$ and a $0.01 \mathrm{kN} / \mathrm{m}$ difference in wall stiffness was found to produce stability without loss of accuracy.

\section{SENSITIVITY ANALYSIS}

The benchmark structure given in Table 1 was used for all sensitivity studies, with one parameter changed at a time.

\section{Effect of Rotational Mass and No Out-of-Plane Walls}

While a greater rotational mass, $J$, causes a decrease in the displacement of the centre of mass, it does not always cause a reduction in the critical wall displacement as shown in Figure 10. Here, for unrealistically low values of rotational mass, the demand increases. However, in general, increasing $J$ decreases the demands and has a significant impact on the response of buildings with no out-of-plane walls.

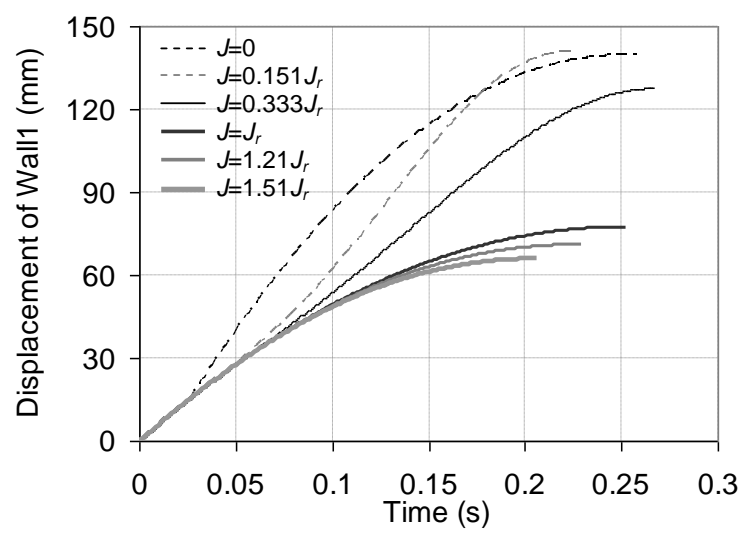

Figure 10: Sensitivity to J (Wall 2 elastic: $S R=\infty$, KRout=1.0).

\section{Effect of Out-of-Plane Wall Stiffness}

Figure 11 shows the response of a structure with Wall 1 yielding, Wall 2 fully elastic, a rotational mass of $0.333 J_{r}$ and with out-of-plane walls of varying stiffness. The out-of-plane wall stiffness has little effect on the centre of mass displacement, but there is a considerable reduction in peak displacement of the critical wall. In addition, the peak critical wall displacement is reached earlier. The reason for this has been described by many authors in the past. It is that out-ofplane walls provide a resisting couple to the twist action; and provided they remain elastic, they are relatively stiff. Therefore, out-of-plane walls provide an effective method for limiting demands on the critical wall.

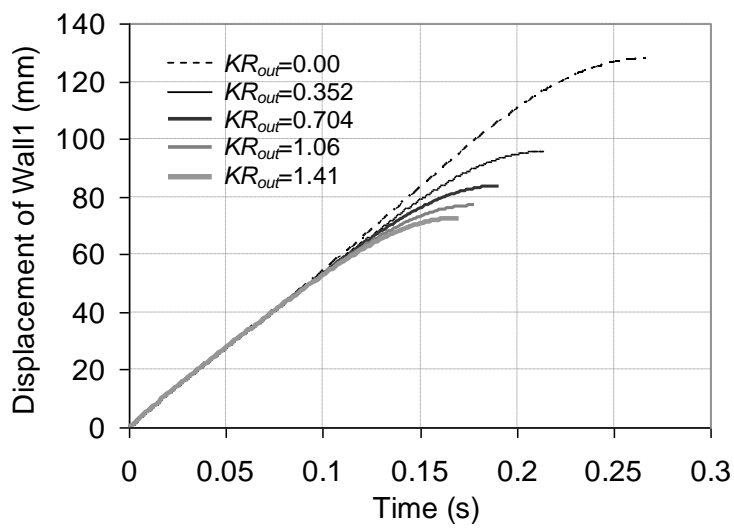

Figure 11: Sensitivity to out-of-plane wall stiffness (Wall 2 elastic: $S R=\infty$ ).

\section{Effect of Wall Strength Difference}

Figure 12 shows that by increasing the strength of Wall 2, a greater torsional response is obtained. That is, unwisely adding strength to a system can be harmful. Thus designers can minimise torsion in structures, undergoing significant yielding, by seeking to reduce strength differences between eccentric lateral-force resisting elements. This is consistent with Paulay's concept of locating the centre of strength as close as possible to the centre of mass [6].

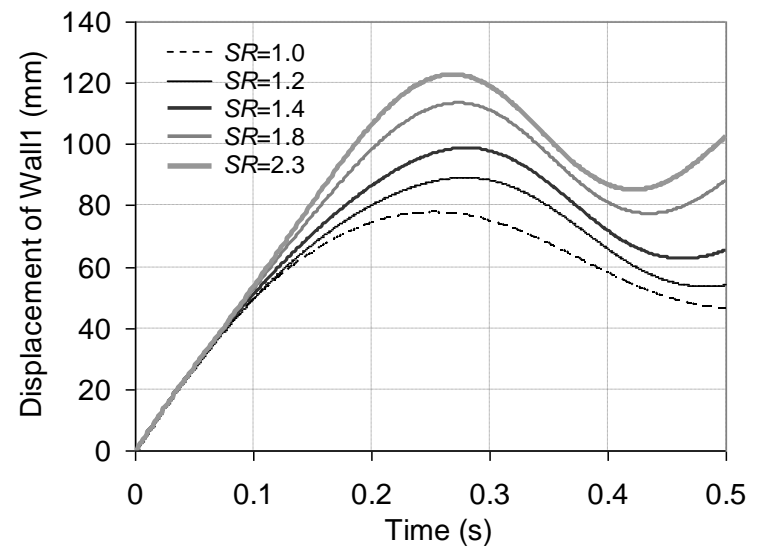

Figure 12: Sensitivity to in-plane wall strength difference.

\section{Effect of Rotational Mass with Out-of-Plane Walls}

Figure 13 shows the impulse time history response for a system with out-of-plane walls, for various rotational masses. Out-ofplane wall stiffness was taken as $7106.1 \mathrm{kN} / \mathrm{m}$, which was half the in-plane wall stiffness $\left(K R_{\text {out }}=0.5\right)$. It may be seen that rotational inertia only has a minor effect on the response and if the out-of-plane wall stiffness is sufficiently high, rotational mass can be ignored. 


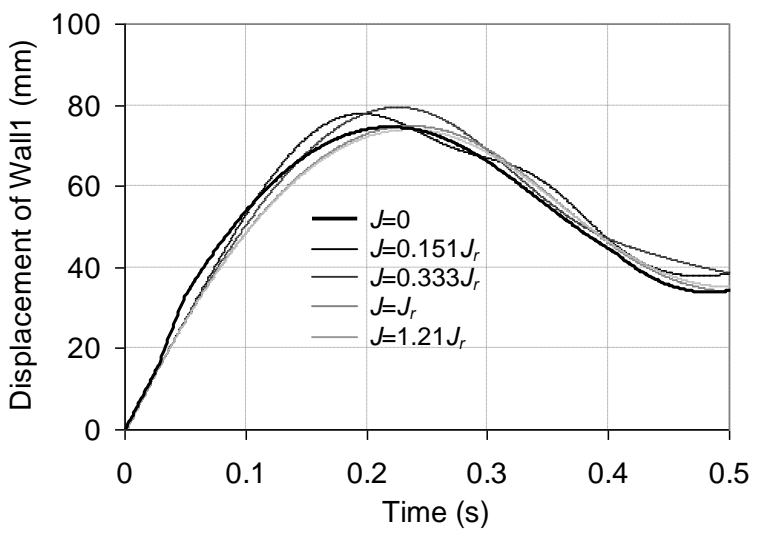

Figure 13: Sensitivity to rotational inertia with out-of-plane walls $($ KRout $=0.5)$.

\section{EARTHQUAKE EFFECT ON CRITICAL WALL DISPLACEMENT}

To evaluate the effect of earthquakes on the response of structures, structures with a number of periods and configurations were analysed. The response of the structure was then compared to the impulse response, using an impulse magnitude which produced the same translational displacement when torsion was restrained.

\section{Methodology with One Record}

To verify that the peak response from an impulse is analogous to the peak response for an actual earthquake, the impulse response was compared to an earthquake time history analysis using the 1994 Northridge Earthquake (Sylmar) NS record.

Analyses were performed using the benchmark structure given in Figure 2 and Table 1 in RUAUMOKO-2D. To approximate the damping in real structures, an initial stiffness Rayleigh damping model was used in the earthquake analysis, with $5 \%$ of critical damping specified in modes 1 and 2 . The fundamental period of the frame was $0.5 \mathrm{~s}$.

Firstly, analysis of the torsionally restrained structure was carried out with the earthquake record to obtain the displacement at the centre of mass as shown in Figure 14. It may be seen that the peak displacement occurs over a very short time so impulse approximation to the response is not unreasonable in this case. An equivalent impulse record was then determined to push the structure to the same peak displacement as that obtained from the earthquake record with the rotational degree of freedom still restrained. The equivalent impulse required to produce this response was a force of $157,000 \mathrm{kN}$ applied over a period of $0.001 \mathrm{~s}$.

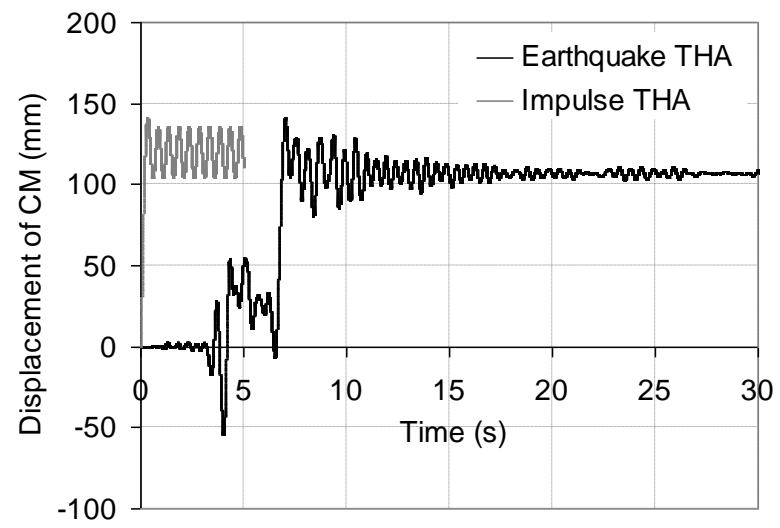

Figure 14: Translational response at centre of mass with torsion prevented.
The earthquake and impulse time history analyses were then performed again with the rotational degree of freedom unrestrained. Figure 15(a) shows that impulse time history gives a $10 \%$ under prediction of the peak critical wall displacement, while Figure 15(b) shows an over-conservative prediction of the peak displacement of the non-critical wall. In general, the impulse response would not be expected to be identical to that of the earthquake.

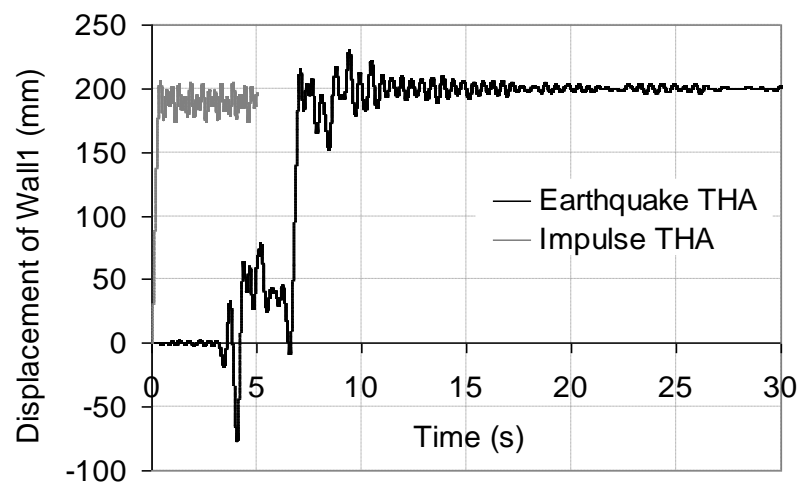

(a) Displacement of Wall 1

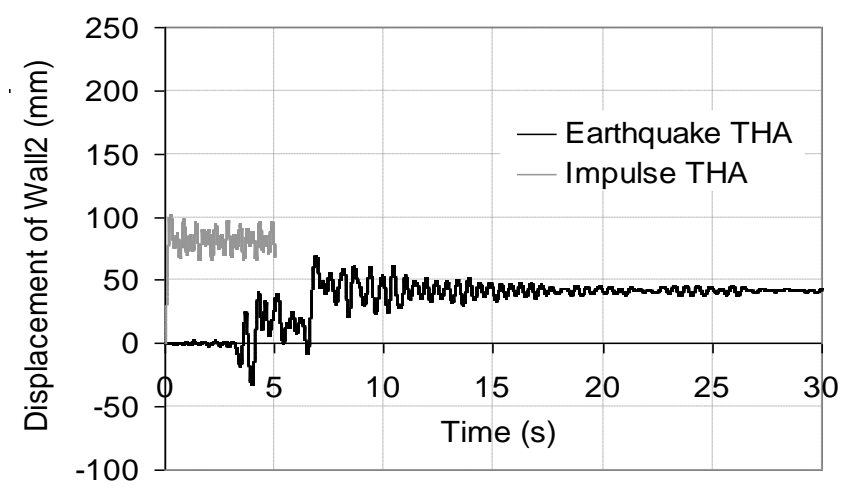

(b) Displacement of Wall 2

\section{Figure 15: Response with Rotational Degree of Freedom} Unrestrained.

\section{Methodology with Several Records}

The single record methodology in (a) above was repeated using the 20 of SAC (SEAOC, ATC, CUREE) ground motion suites for Los Angeles on structures with different configurations to statistically quantify the difference between predicted displacements from earthquake time history analyses and nonlinear impulse procedures. Simulations were run using Mathworks MATLAB in conjunction with RUAUMOKO-2D to allow the analyses to be automated.

The benchmark structure was reused and altered, changing one parameter at a time to give different configurations. Parameters addressed were: excitation scale factors for both the impulse and earthquake analyses to produce ductility demands of $1,2,5$ and 8 in the critical wall when torsion is restrained; various masses to achieve periods of $0.1,0.3,0.5,1,2$ and 3 seconds; rotational masses of $0,0.5 J_{r}, J_{r}$ and $1.5 J_{r}$; wall strength ratios of $1,1.2,1.36,2,3,5$ and 8 ; out-of-plane wall stiffness ratios of $0,0.1,0.5$ and 1 ; and lastly, in-plane wall stiffness ratios of $0.5,0.75,1$ and 1.25 .

Impulse and earthquake scale factors were found by iteration using the bisection method prior to each $2 \mathrm{DOF}$ time history analysis. Iteration continued until the desired translational (1DOF) ductility was achieved within a $1 \%$ tolerance. The subsequent $2 \mathrm{DOF}$ simulation allowing twist was carried out 
using the scale factor previously found to obtain the peak critical wall displacement. The results are plotted as a ratio of peak earthquake displacement to peak impulse displacement in Figure 16. The median \pm dispersion lines represent the 16 th and 84th percentile values found by fitting a log-normal distribution.

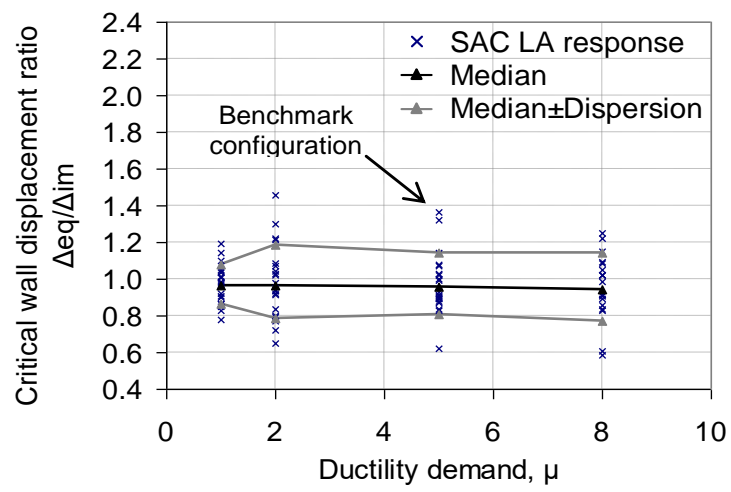

(a) Variable: Ductility demand

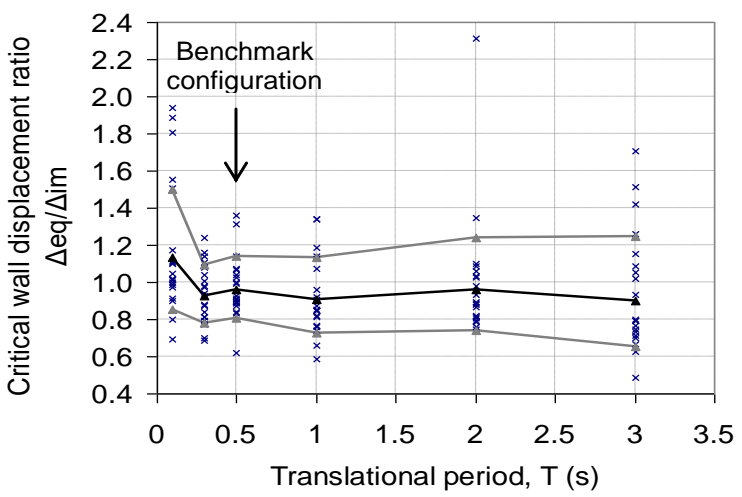

(b) Variable: Translational period

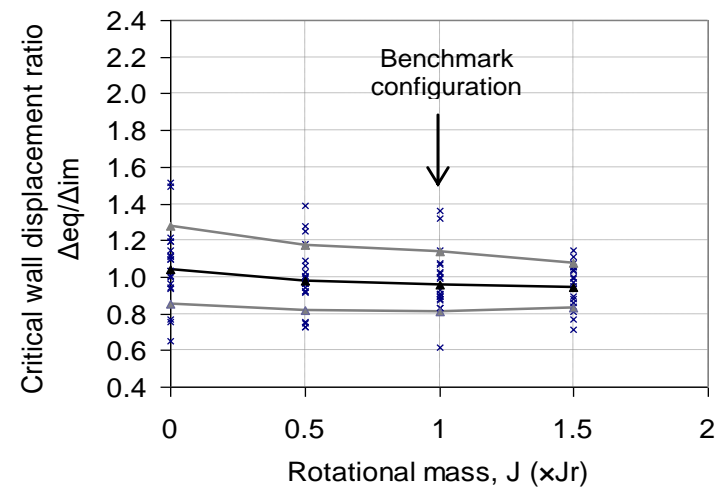

(c) Variable: Rotational mass

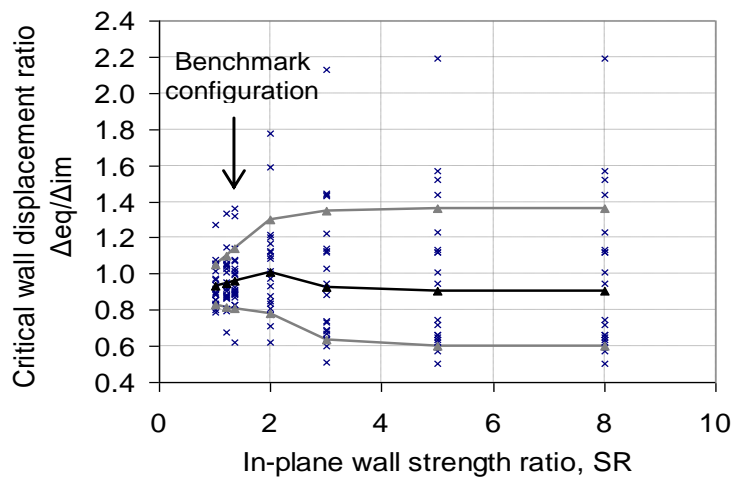

(d) Variable: Wall strength difference

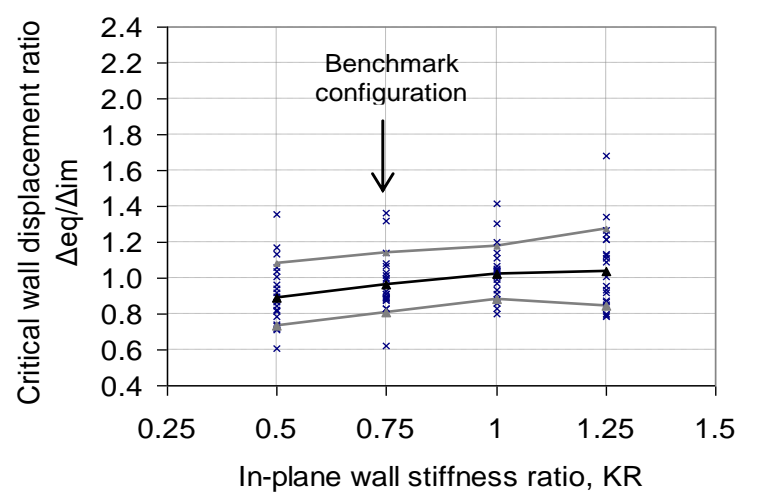

(e) Variable: Wall stiffness difference

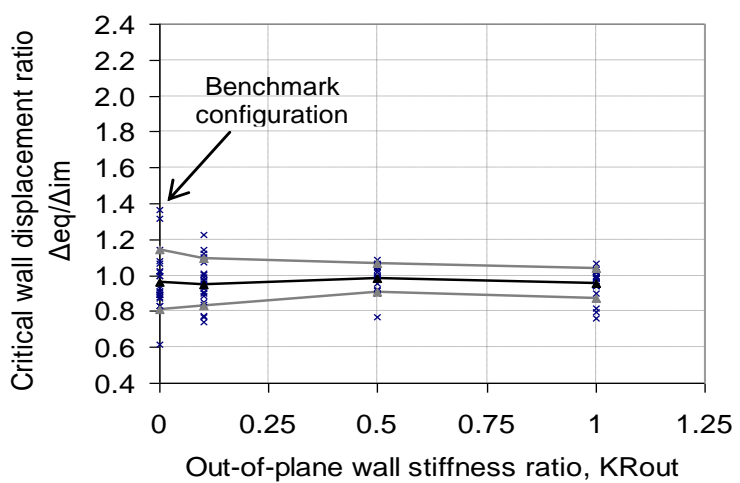

(f) Variable: Out-of-plane wall stiffness

Figure 16: Ratio of peak SAC ground motions response to peak NIP response for Benchmark structure: $\mu=5, T=0.5 \mathrm{~s}$, $J=J r, S R=1.36, K R=0.75$ and $K R o u t=0$.

Table 2 summarises the average median displacement ratio and dispersion for each variable that was altered. Overall, the nonlinear impulse procedure provided a good prediction of peak critical wall displacement with dispersions ranging between 0.12 and 0.27 . The overall critical wall displacement ratio had a median of 0.96 and dispersion of 0.19. Based on a log-normal distribution, the overall median and dispersion can be used to amplify results from NIP to give a final prediction of peak earthquake response with a specified statistical level of confidence. This is shown in the following design approach.

Table 2: Average ratios of peak earthquake response to peak impulse response, for different variables based on the lognormal distribution.

\begin{tabular}{ccccc} 
& Median & $\begin{array}{c}\text { Dispersion } \\
(\boldsymbol{\sigma l n} \mathbf{x})\end{array}$ & $\begin{array}{c}\mathbf{1 6}^{\text {th }} \\
\text { percentile }\end{array}$ & $\begin{array}{c}\mathbf{8 4}^{\text {th }} \\
\text { percentile }\end{array}$ \\
\hline$\mu$ & 0.96 & 0.17 & 0.81 & 1.14 \\
$\mathrm{~T}$ & 0.96 & 0.24 & 0.76 & 1.23 \\
$\mathrm{~J}$ & 0.98 & 0.17 & 0.83 & 1.17 \\
$\mathrm{SR}$ & 0.94 & 0.27 & 0.72 & 1.24 \\
$\mathrm{KR}$ & 0.98 & 0.18 & 0.82 & 1.17 \\
$\mathrm{KR}$ & 0.96 & 0.12 & 0.85 & 1.08 \\
\hline Overall & 0.96 & 0.19 & 0.77 & 1.16 \\
\hline
\end{tabular}




\section{DESIGN APPROACH}

\section{Methodology}

The following step by step methodology is proposed for structures of the type described above, which are subject to earthquake excitations.

Step 1. Estimate the likely displacement response of the structure using standard methods assuming that no twist occurs about the vertical axis, $\Delta_{\text {NoTwist }}$.

Step 2. Find the impulse, $F \Delta t$, that would push the structure to $\Delta_{\text {NoTwist }}$, if twist is restrained and no damping is assumed, using the 2DOF methodology developed above to model the structure. This may be carried out using a very high value for $k_{r}$.

Step 3. Use $F \Delta t$ to obtain the response of the critical wall, $\Delta_{T w i s t}$, if twist about the vertical axis is not restrained, using the 2DOF methodology to model the structure. A realistic $k_{r}$ value should be used.

Step 4. Obtain the ratio of earthquake displacement to impulse displacement from the previous section, $R_{E \mid I}$ for the desired statistical level of confidence.

Step 5. Multiply $\Delta_{T w i s t}$ by $R_{E \mid I}$ to estimate the demand in the critical element.

\section{Example}

Step 1. For the frame given in Figure 17, the fundamental period with no torsion is $0.7 \mathrm{~s}$. It is subject to an earthquake which produces a total displacement of $\Delta_{\text {NoTwist }}=60 \mathrm{~mm}$ when twist about the vertical axis is restrained. Any method satisfying code requirements can be used for this displacement prediction.

Step 2. By trial and error, the impulse, $F \Delta t$, that would push the structure to $\Delta_{\text {NoTwist }}$ is a force of $165,000 \mathrm{kN}$ acting for $0.001 \mathrm{~s}$.

Step 3. When the structure is permitted to twist about its vertical axis, under this impulse, the response of the critical wall, $\Delta_{T w i s t}$, is found to be $83 \mathrm{~mm}$. This is a $38 \%$ increase due to torsion.

Step 4. The ratio of earthquake displacement to impulse displacement from Table 2, $R_{E \mid I}$, for an overall $84 \%$ statistical level of confidence is 1.16 .

Step 5. The estimated displacement demand on the critical element is therefore $1.16 \times 83 \mathrm{~mm}=96 \mathrm{~mm}$. This corresponds to a ductility demand of 6 .

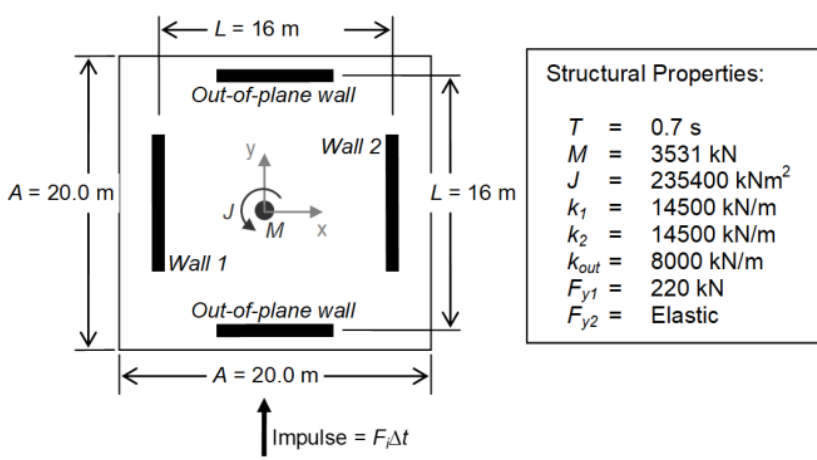

Figure 17: Plan and properties of structure.

\section{CONCLUSIONS}

Analyses of single story structures were carried out to evaluate the effect of torsion. It was found that:

1. Impulse loading allows the dynamic response of a structure to be quantified, including rotational inertia effects. For no rotational mass, results were the same as those predicted by Paulay [4]. Nonlinear Impulse Procedure was shown to be a valuable tool for understanding the dynamic response of systems

2. A closed-form solution of the single-storey system subject to impulse loading was developed based on 2DOF freevibration concepts. This solution was accurate in most cases, but in some cases conservatively predicted the peak response.

3. Consistent with previous literature, larger in-plane wall strength differences increase the torsional response, while out-of-plane wall stiffness and rotational mass decrease the response. However, for realistic values of out-of-plane wall stiffness, the solution is not very sensitive to the effect of rotational mass and neglecting the rotational mass effect is not unreasonable.

4. From analyses using real earthquake records, NIP was found to give good predictions of peak critical wall response. From this understanding, a design procedure is developed for a specified level of confidence and a design example is provided.

\section{ACKNOWLEDGMENTS}

The authors are grateful to Professor Athol Carr for his valuable help with the computer modelling and to the NZ Earthquake Commission for funding research in structural regularity, to which this work contributes.

\section{REFERENCES}

1. Rosenblueth E and Elorduy J (1969). "Responses of Linear Systems to Certain Transit Disturbances". Proceedings of Fourth WCEE, Santiago, January 13-18, 1 (A-1), 185-196.

2. Rutenburg A (2002). "EAEE Task Group (TG) 8: Behaviour of Irregular and Complex Structures Asymmetric Structures - Progress Since 1998". Proceedings of the 12th European Conf. on Earthquake Engineering, London, September 9-13, \#832.

3. De Stefano M and Pintucchi B (2006). "EAEE Task Group (TG) 8: Seismic Behaviour of Irregular and Complex Structures: Progress Since 2002". Proceedings of the 1st European Conf. on Earthquake Engineering and Seismology, Geneva, September 3-8, \#1443.

4. Paulay T (1996). "Seismic Design for Torsional Response of Ductile Buildings". Bulletin of the NZ Society for Earthquake Engineering, 29(3): 178-198.

5. Paulay T (1998). "Torsional Mechanisms in Ductile Building Systems". Earthquake Engineering and Structural Dynamics, 27: 1101-1121.

6. Paulay T (2001). "Some Design Principles Relevant to Torsional Phenomena in Ductile Buildings". Journal of Earthquake Engineering, 5(3): 273-308.

7. Castillo R (2004). "Seismic Design of Ductile Asymmetric Structures". PhD Dissertation, University of Canterbury, Christchurch, New Zealand, pp.354.

8. Myslimaj B and Tso WK (2004). "Desirable Strength Distribution for Asymmetric Structures with StrengthStiffness Dependent Elements". Journal of Earthquake Engineering, 8(2): 231-248.

9. Trombetti T, Gasparini G and Silvestri S (2002). "A New Simplified Approach to the Analysis of Torsional Problems in Eccentric Systems: the "Alpha" Method". Proceedings of Third European Workshop on the Seismic Behaviour of Irregular and Complex Structures, Firenze, September 1617.

10. Sommer A and Bachmann H (2005). "Seismic Behaviour of Asymmetric RC Wall Buildings: Principles and New 
Deformation-Based Design Method". Earthquake Engineering and Structural Dynamics, 34: 101-124.

11. Pettinga JD, Pampanin S, Christopoulos C and Priestley MJN (2007). "Developments in the Prediction and Mitigation of Residual Deformations due to Seismic Demand, Including Asymmetric Structural Response". Research Report No. ROSE 2007/01, European School for Advanced Studies in Reduction of Seismic Risk, Pavia, pp.295.

12. Fajfar P and Fischinger M (1996). "The N2 Method for the Seismic Damage Analysis of RC Buildings". Earthquake Engineering and Structural Dynamics, 25: 31-46.

13. Fajfar P, Marusic D and Perus I (2005). "The N2 Method for Asymmetric Buildings". Proceedings of the First
European Conference on Earthquake Engineering and Seismology, Geneva, September 3-8.

14. Beyer K (2008). "Seismic Design of Torsionally Eccentric Buildings with U-shaped RC Walls". Research Report No. ROSE 2008/0X, European School for Advanced Studies in Reduction of Seismic Risk, Pavia, pp.343.

15. Au EV (2007). "Simplified Methodology for the Seismic Response of Torsionally Irregular Structures". Third Professional Project Report, Department of Civil Engineering, University of Canterbury, Christchurch, New Zealand, pp.79.

16. Carr AJ (2004). "Ruaumoko". Department of Civil Engineering, University of Canterbury, Christchurch. 